\title{
Manipulating Spectra with DTSA-II
}

\section{Nicholas W. M. Ritchie}

National Institute of Standards and Technology, Gaithersburg, MD 20899-8371

nicholas.ritchie@nist.gov

\section{Introduction}

The previous article in this series [1] introduced DTSA-II and provided some background information including how to configure DTSA-II for your $\mathrm{x}$-ray detector and how to read spectra from disk. This article presents more basic background material. It discusses how to display and perform basic manipulations on spectra including reading the contextual properties of spectra, visualizing spectra, labeling characteristic x-ray lines, and exporting the results as publication-quality graphics. You are encouraged to follow along using DTSA-II and example spectra, which may be downloaded for free from http://www.cstl.nist.gov/ div837/837.02/epq/dtsa2/index.html or equivalently http:// goo.gl/MI1Ku. With this background material, we will be ready to consider more sophisticated tasks like spectrum quantification and simulation in upcoming articles.

\section{Displaying Spectra}

Spectrum display panel. As discussed in the previous article, DTSA-II supports the reading of many different kinds spectral formats, including the ISO standard EMSA file format [2]. The number of spectra that DTSA-II can have open simultaneously is only limited by available memory. though because all modifications to cleared spectra will be lost unless the modified spectra have been written to disk.

Spectrum properties panel. To the right of the spectrum list is the spectrum properties panel (see Figure 2). When one spectrum is selected in the spectrum list, the spectrum properties panel displays all the properties associated with the selected spectrum. These properties include those properties read from the spectrum file and those properties applied from the default detector. (See the previous article for a description of the default detector [1].) In addition, there may be properties that are added as the spectrum is manipulated within DTSA-II. One such example is the "Duane-Hunt" limit property. The Duane-Hunt limit, an estimate of the effective electron beam accelerating potential, is calculated when the spectrum is read from disk. If the Duane-Hunt limit is substantially less than the nominal beam energy, it suggests that the sample is charging. Other properties that can be added by DTSA-II include the "microanalytical composition," the "standard composition," and the "k-ratio set." If the microanalytical or standard composition is defined, then these are displayed in the lower right corner of the spectrum tab in the composition table panel.

A full list of open spectra is displayed in the spectrum list on the left edge of the spectrum utility panel (see Figure 1). You may select up to 50 open spectra to display in the spectrum display panel. To select multiple spectra, you can hold down either the shift key (contiguous selection) or the control key (discontiguous selection) while clicking on the name of the spectrum.

Clicking the right mouse button while the cursor hovers over the spectrum list panel will display a context menu containing actions to "select all," "select none," or "clear selected." The first two items modify which spectra are highlighted and thus which spectra are displayed in the spectrum display panel. The last item removes spectra from the spectrum list and clears them from memory. Be careful

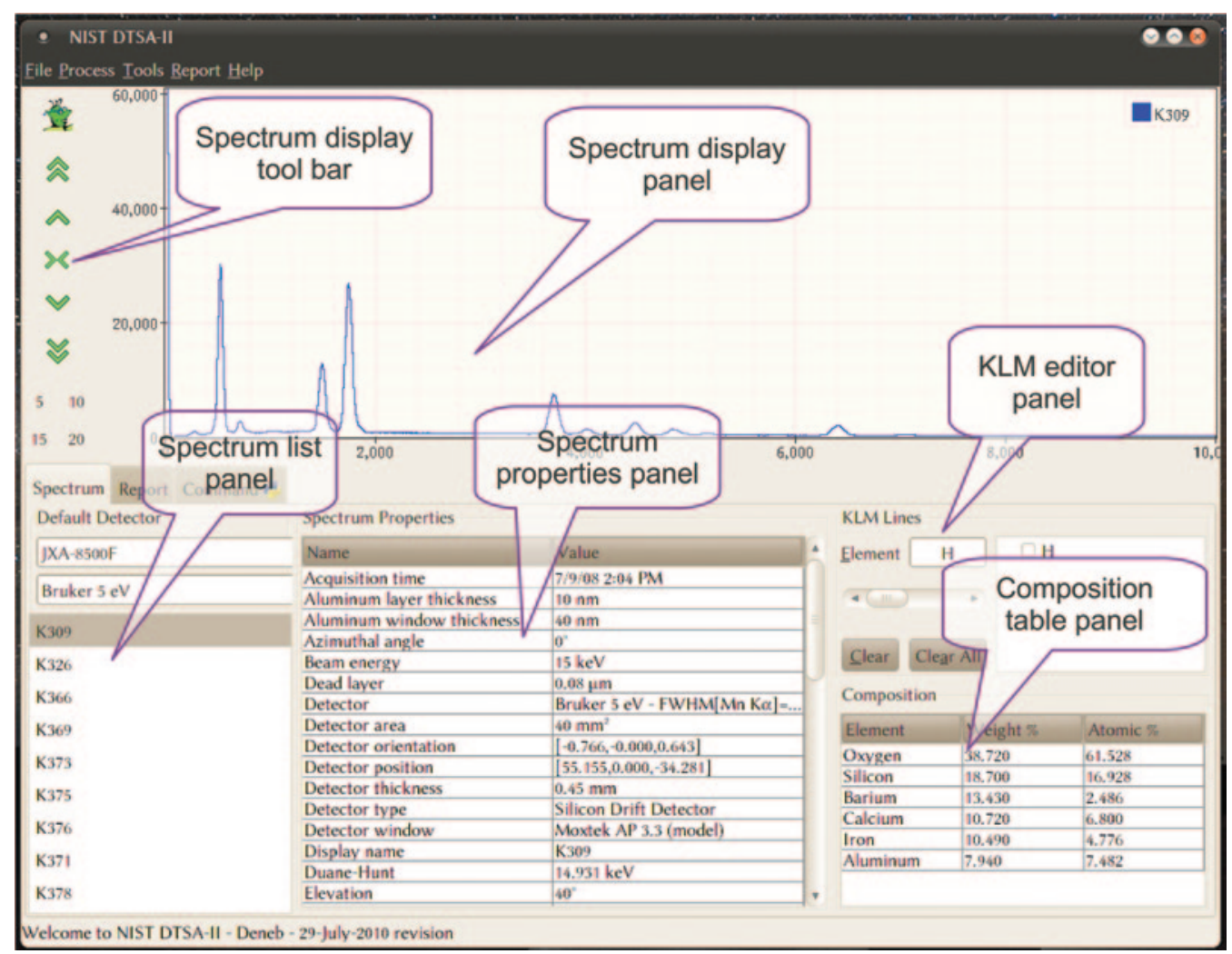

Figure 1: The primary panels used to manipulate spectra. 


\section{mniprobe}

EXTENDING FIB \& SEM CAPABILITIES

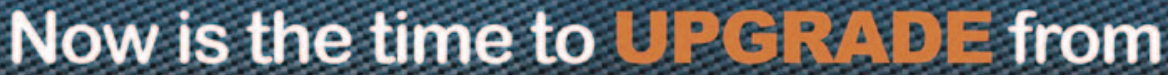 Basic to Automated in situ Lift-out}

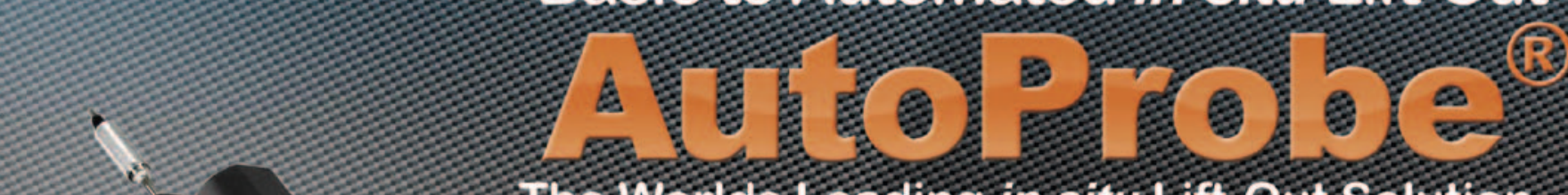

The Wordos seading in situ 5 ift - Out Solution

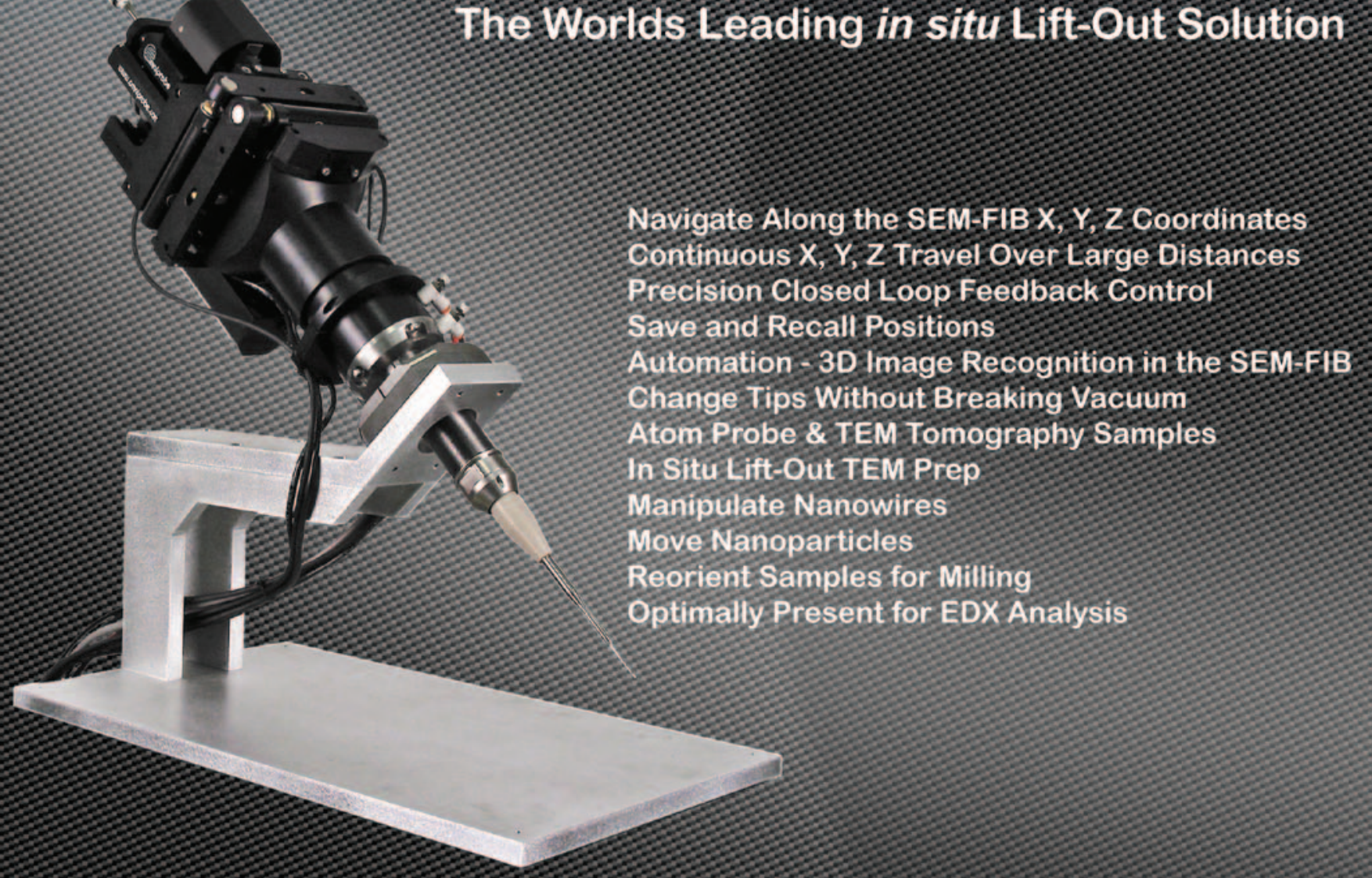

UPGRADE your Model 100.7 Value Probe Nanomanipulator to an AUTOPROBE ${ }^{\circledR}$

\section{Upcoming Shows}

\section{Semicon Mar 15-17. Shanghai, China}

IUMAS-V May 22-26 - Seoul, Korea

EIPBN May 31-June 3-Las Vegas, NV

IPFA Jul 5-7-Inchean, Korea

M\&M Aug 7-11-Nashville, TN

Microscopy Conference 2011 Aug 28 -Sep 2-Kiel, Germany

AMFA Aug 29-Boston, MA

ISTFA Nov 15-17 - San Jose, CA

MRS Fall Nov 28-Dec 2-Boston, MA 


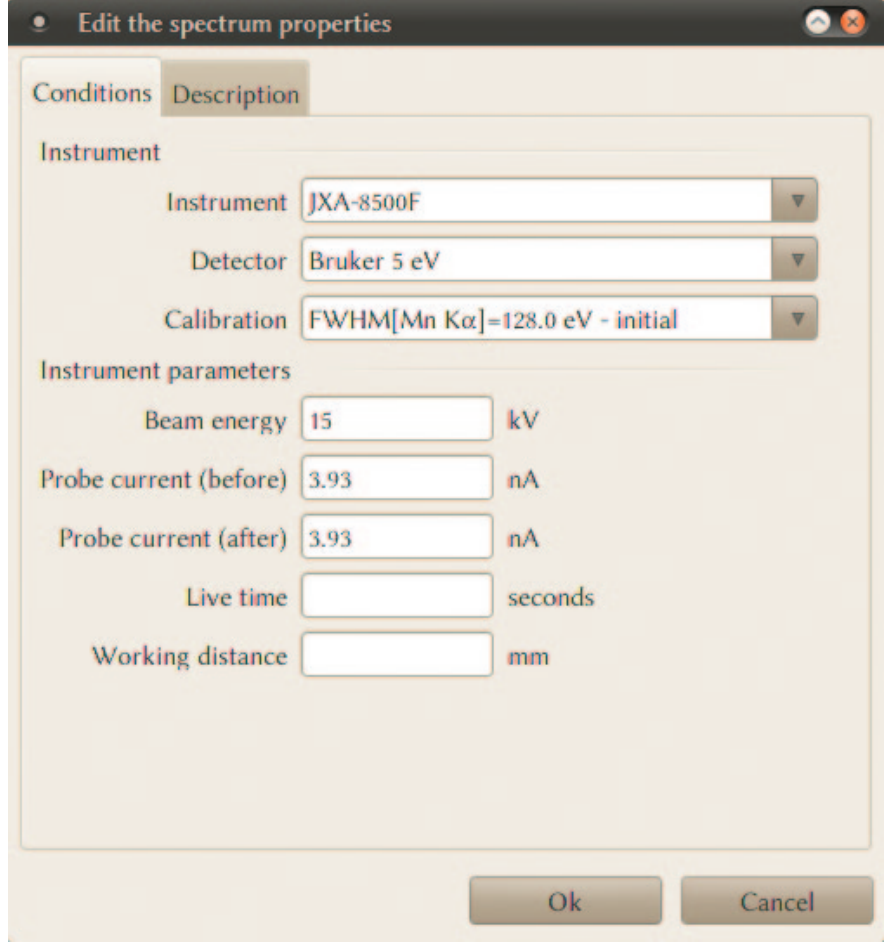

Figure 2: The spectrum properties dialog. This dialog permits editing the properties of one or more spectra.

If multiple spectra are selected simultaneously, then the behavior of the spectrum property panel changes. The panel only displays the subset of properties that all the selected spectra share in common. For example, the "beam energy" property will display if all the selected spectra were collected at the same beam energy. This behavior permits you to review the properties common to multiple spectra.

Modifying spectrum properties. Many of the spectrum properties can be modified on a spectrum-by-spectrum basis or in batch mode. Select one or more spectra that you wish to modify in the spectrum list panel. Then select the "tools $\rightarrow$ edit spectrum properties" menu item. The spectrum properties dialog will display. Through this dialog you can associate the spectra with different detectors or edit the beam energy, probe current (both before and after acquisition), live time, working distance, and other descriptive properties. Only those values that you actively modify will be updated when you select "ok" to close the dialog. The same modifications will be made simultaneously to all selected spectra. Please note that only the in-memory copy of the spectrum is changed. To record the changes, you must save the spectrum to disk.

\section{The Material Editor}

Specifying materials. Spectra from materials of known composition are often used to quantify spectra from unknown materials. The user must specify a description of the known material with the spectrum. This can be accomplished by selecting one or more spectra in the spectrum list then selecting the "tools $\rightarrow$ assign material" menu item. The material editor dialog is used in many different contexts and, despite its simple appearance (see Figure 3), is really quite sophisticated.
Materials are described within DTSA-II by their composition and density. The composition can be defined on an element-by-element basis in terms of weight fraction or atomic fraction. The editor allows you to enter compositions using either format and to transform between the two composition formats.

Stoichiometric materials. For stoichiometric compounds, the quickest way to enter materials is to enter a chemical formula into the "name" field and then press the button with the magnifying glass icon. The formula will be parsed and the correct composition automatically entered. For example, entering "Al2O3" will produce a material $\left(\mathrm{Al}_{2} \mathrm{O}_{3}\right.$ / alumina) with 47.07 percent by weight $\mathrm{Al}$ and 52.93 percent by weight $\mathrm{O}$. For the parser to work, the element abbreviations must be capitalized correctly, and the formula must be unambiguous. The parser can handle complex formulas like "Ca5(PO4)3F" for fluorapatite where the PO4 term is taken three times for a total of $5 \mathrm{Ca}$ atoms, $3 \mathrm{P}$ atoms, $12 \mathrm{O}$ atoms, and $1 \mathrm{~F}$ atom per unit cell.

Non-stoichiometric materials. If the material is not stoichiometric (for example, glasses or alloys), it is possible to enter the composition element-by-element using the "element" and "quantity" edit boxes and the "add" button. If the editor contains incorrect information, you can use the "clear" button to eliminate it all or the "delete" button to remove individual elements. The format for the quantity data depends on whether the "mode" is "weight fraction" or "atomic proportion." If the mode is weight fraction, enter the amount of each element as a percentage by weight of the total mass. If the mode is atomic proportion, enter for each element the number of atoms per unit cell.

Regardless of how you enter the composition, each material can be assigned a user-friendly name like "Fluorapatite" and a

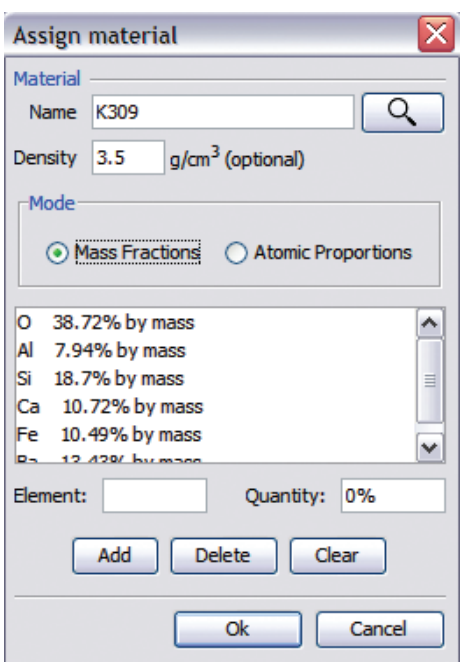

Figure 3: The material editor dialog. This dialog allows you to specify the composition and density of the material from which a spectrum was collected. The dialog is backed by a database, which remembers the definition of the materials indexed by their name for recall later. density in grams per cubic centimeter. Not all uses of the material property require a density, but it is often a good idea to enter the correct density when available.

Rather than forcing the user to enter material data afresh each time, the material editor dialog is backed by a database. The material database is indexed by material name. If the database contains a material, then you can enter the name into the dialog's name edit window and press the tab button or the magnifying glass button. The dialog will search the database for a material name that matches (exact, 
case sensitive). If one is found, the composition and density will be retrieved from the database and automatically entered into the dialog.

As you define new materials, they are automatically and transparently added to the database. Thus, it is best to carefully assign a user-friendly name and a density for each material because it facilitates reuse. In time you will build up a substantial and useful database of materials. If you modify the definition (composition or density) of an existing material, the program will ask whether you want to update the database definition.

\section{Manipulating the Spectrum Display}

The spectrum display (see the top of Figure 1) is a plotting tool specialized for displaying $\mathrm{x}$-ray spectra. It is capable of displaying up to 50 spectra simultaneously. Energy-dispersive $\mathrm{x}$-ray spectra are a histogram of $\mathrm{x}$-ray counts in bins of increasing energy. The horizontal axis represents $\mathrm{x}$-ray photon energy measured in electron volts $(\mathrm{eV})$ and is divided into equal-sized bins. On most spectrometers these bins are nominally either $10 \mathrm{eV} /$ bin wide (most common) or $5 \mathrm{eV} /$ bin wide. The vertical axis represents the count of $\mathrm{x}$-ray events falling within an energy bin. If you examine a reduced range of energies, then you will notice that the spectrum is not a continuous curve but is represented by a series of steps.

Spectrum display toolbar. To the left of the spectrum display is the spectrum display tool bar with buttons to manipulate the vertical and horizontal scales. The top button (曐) autoscales the axes to display all spectral data above $0 \mathrm{eV}$. The vertical scale is set to display the highest peak (excluding the zero strobe peak). The vertical arrow buttons modify the vertical scale. The double arrows step by a factor of 5 and the single arrows by a factor of 2 . In addition to the graphical buttons, a mouse equipped with a wheel can be used to scroll the vertical axis. When the cursor is located over the spectrum display panel, rolling the mouse wheel will increase or decrease the vertical axis.

Horizontal scale changes. The horizontal axis can be modified using the buttons labeled " 5 ," " 10 ," " 15 ," or "20." These buttons set the horizontal axis range to $0 \mathrm{eV}$ to $5 \mathrm{keV}$, $0 \mathrm{eV}$ to $10 \mathrm{keV}, 0 \mathrm{eV}$ to $15 \mathrm{keV}$, or $0 \mathrm{eV}$ to $20 \mathrm{keV}$, respectively. It is possible to zoom in to different energy ranges using the energy region cursor and the doubly-opposed horizontal arrows (囚) button. Energy regions can be specified by left-clicking and dragging the mouse cursor within the spectrum plot panel. The selected regions will display in blue while the drag is in process and in yellow when the left mouse button is released. You may select multiple regions by repeating the drag-and-click process. Be careful not to double-click in the plot panel because this engages the single channel cursor (a vertical blue line). If you do engage the single channel cursor, a second double-click will disengage it. Once one or more ranges of channels have been selected, the horizontal arrow button ( $\mathbb{X}$ ) will zoom in to a range of energies, which minimally encompasses all selected energy ranges.

It is also possible to perform many of the same actions using the spectrum display panel's context-sensitive menu. A

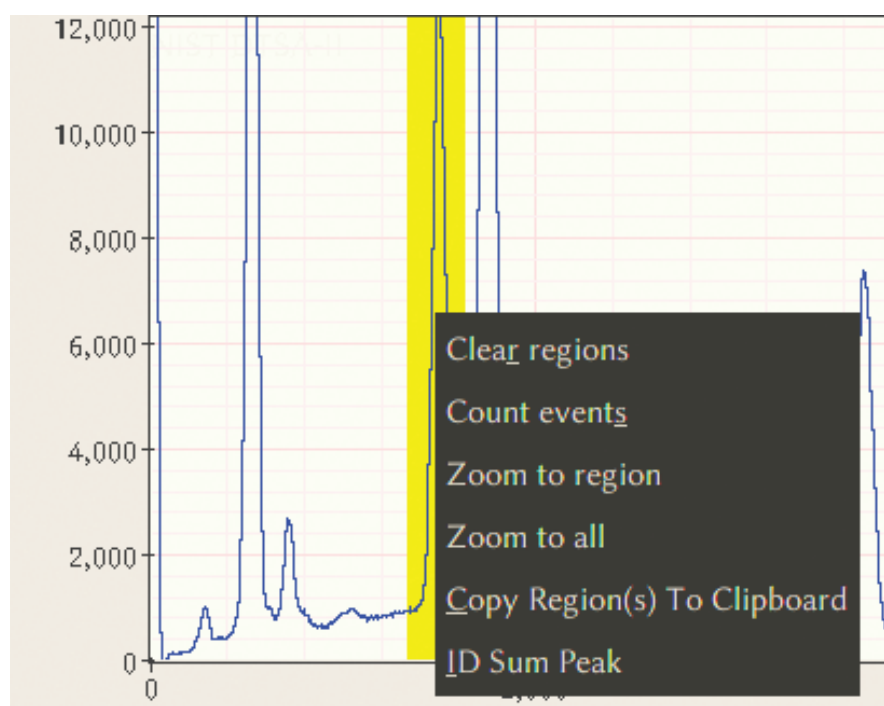

Figure 4: The energy region menu. This menu is accessed via a right mouse click over a yellow highlighted region of spectrum. It contains actions to be performed on the highlighted range of channels. Highlighted regions are created by clicking and dragging over a range of energies.

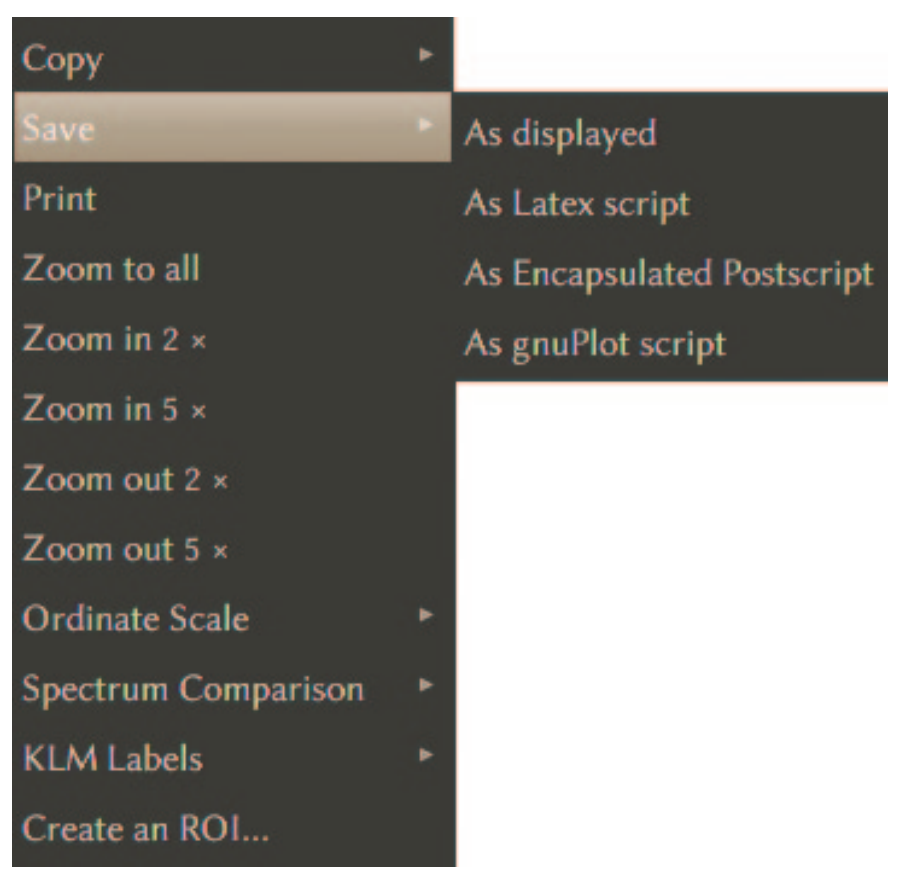

Figure 5: The main spectrum display menu. This menu contains actions to export and configure the spectrum display. The "copy $\rightarrow$ as bitmap" menu action places a low-fidelity bitmap of the spectrum display on the system clipboard. The "save $\rightarrow$ as displayed" action exports a high-fidelity representation of the spectrum display to a file.

right mouse click over the spectrum display panel will display one of two different menus. If the right click is over a highlighted (yellow) region, then a menu with actions related to the energy region will display (see Figure 4). If the right click is not over a highlighted region, then a menu with generic spectrum manipulation options will display (see Figure 5). Many of the same zoom options are available from this menu. In addition, one menu item allows you to explicitly specify ranges of channels using a dialog box to enter the energies as text values. 
This dialog box has a zoom button, which immediately reduces the energy axis range to the specified values.

Vertical scale changes. The generic spectrum display panel menu also has menu items to change the vertical (ordinate) scale mode from the default linear axis to logarithmic or square root axes. The linear vertical axis is the most common display option, but the logarithmic axis is often better for viewing spectra with major, minor, and trace elements. The square root axis takes into account the Poisson (count statistics limited) nature of EDS spectra to display the spectra such that the relative magnitude of the bin-to-bin or channel-to-channel variation looks similar for both high and low count features. DTSA-II recalls the previous setting of the axis scale mode between program invocations.

Relative vertical display. The generic spectrum display panel menu also has menu items to specify how the spectra are scaled relative to each other. The most common display mode is to plot the spectra on the "same scale." In this case, the labels on the vertical axis represent the number of x-ray event counts. Sometimes it is convenient to scale several spectra relative to some characteristic they hold in common. There are various different options. One option is to plot all spectra relative to the tallest peak in the spectrum (ignoring any peaks in the range of channels identified by the relevant detector's zero strobe peak). Because there is no consistent mapping between the vertical axis and counts, the vertical axis is unlabeled. Another option is to scale the spectra relative to an electron dose of $60 \mathrm{nA} \cdot \mathrm{s}$. In this case, the vertical axis is labeled with the $\mathrm{x}$-ray counts normalized to a relative dose of $60 \mathrm{nA} \cdot \mathrm{s}$. (For this operation, spectra for which these quantities are not available are assumed to have a probe current of $1 \mathrm{nA}$ and a live time of $60 \mathrm{~s}$.)

Alternatively, the spectra can be scaled relative to the number of counts integrated over a range of energies. There are two alternatives. Either you can specify one or more ranges of channels over which to integrate, by dragging-and-clicking to select ranges of energies, or you can integrate over all energies. The "scale to region integral" employs the user-specified range(s). The "scale to equal integral" scales relative to the integral of all counts in the spectra.

\section{Characteristic Line Labels}

DTSA-II contains a database of characteristic line energies and approximate line weights for all elements up to $Z=95$. Characteristic line labels are often called "KLM labels" because the characteristic $\mathrm{x}$-ray lines are associated with the $\mathrm{K}, \mathrm{L}$, and $\mathrm{M}$ atomic shells. The $\mathrm{K}$ lines are the most energetic $\mathrm{x}$-rays, and the $\mathrm{M}$ lines are the least energetic $\mathrm{x}$-rays for a given element. Assuming the electron beam can excite them, $\mathrm{K}$ lines are typically visible for all but the lowest $\mathrm{Z}(<\mathrm{Be})$ and the highest $\mathrm{Z}$ elements. $\mathrm{L}$ lines are visible for elements above about $\mathrm{Ca}$ and $\mathrm{M}$ lines for elements above $\mathrm{Ba}$.

The K, L, and M series are families of characteristic $\mathrm{x}$-ray lines, each line uniquely identified with a transition between two bound levels in an atom. The most intense $\mathrm{K}$ line is nominally the K-L3 line (representing a transition from the L3 shell to the $\mathrm{K}$ shell), and the line is designated the Kal. The K-L3 notation is more modern and is the preferred notation according to the IUPAC [3]. The archaic Kal notation (Siegbahn notation) is preferred by most long-term practitioners. DTSA-II supports both. The default spectrum display pop-up menu allows you to select between "IUPAC," "element," "large font" (element only), "Siegbahn," or "no labels."

KLM lines panel. Characteristic lines are selected using the KLM lines panel on the Spectrum tab (see Figures 1 and 6). This panel provides controls to select the full range of elements, and, for each element, select either full families of lines $(K$, $\mathrm{L}$, and/or $\mathrm{M})$, common subsets of lines (Ka, $K \beta, \mathrm{L} \alpha$, etc.), or individual lines (K-L3, L3-M5, M5-N7, etc.).

Element selection. There are a number of ways to select an element: (a) You may enter the full name or the standard abbreviation or the atomic number into the element edit box, (b) You may use the scroll bar located below the element edit box, (c) You may locate the mouse cursor within the KLM panel (except the tree view) and rotate the mouse wheel.

When the element changes, the list of available transitions will update in the KLM panel tree view. In addition to KLM lines, the spectrum display panel can display markers at the energies associated with atomic shell edges (absorption edges) and at the positions of the Si escape peaks. The Si escape peak

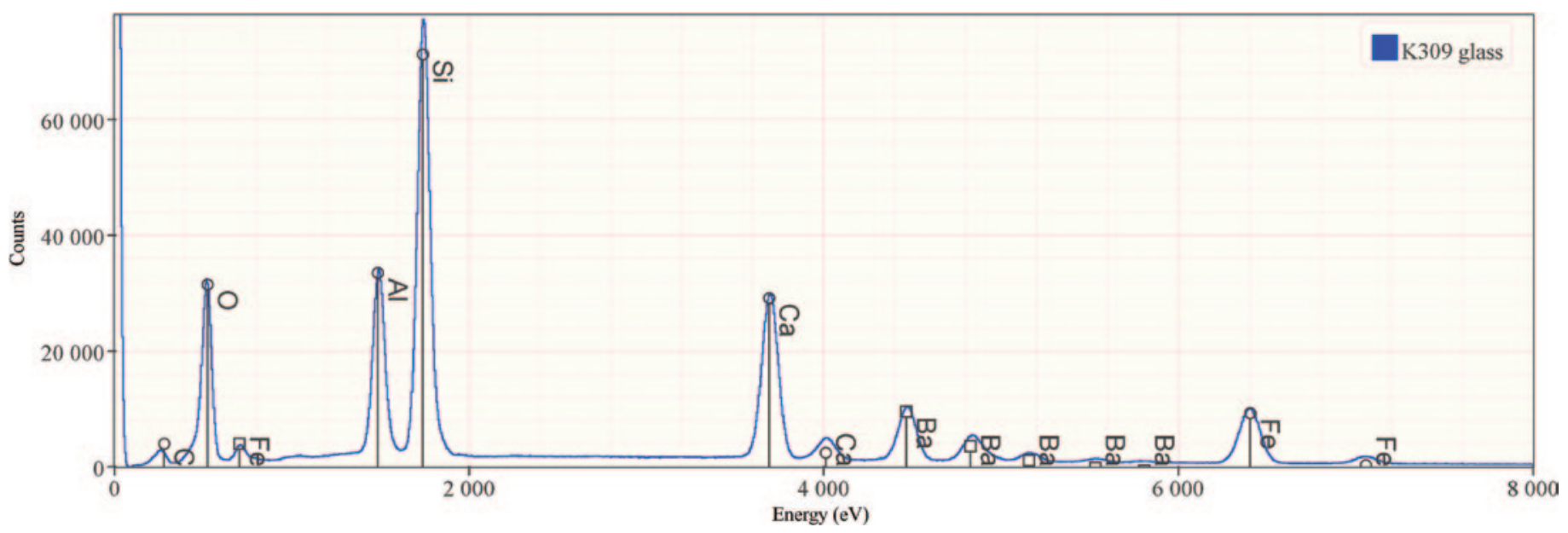

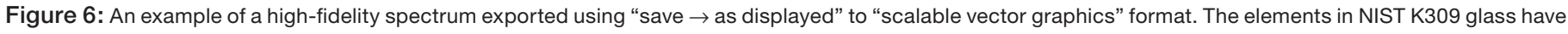
been annotated with IUPAC labels. 
is a common EDS spectral artifact that occurs exactly one Si K $\mathrm{x}$-ray energy (1.74 keV) below sufficiently energetic x-rays.

Special peaks. The KLM tree view allows you to select characteristic transitions, absorption edges, and silicon escape peaks. The tree view interface allows you to select groups of lines quickly yet also permits selecting or deselecting individual lines. Transitions are subdivided into major lines and minor lines to mitigate line clutter. Furthermore, if the cursor is located in the element edit box, keyboard shortcuts can be used to select sets of lines. "Alt-K," "Alt-L," and "Alt-M" select the K, L, and $\mathrm{M}$ families, respectively. "Alt-S" selects $\mathrm{Si}$ escape peaks, and "Alt-E" selects absorption edges. Using the mouse wheel and the keyboard, it is easy to label spectra with many elements.

One additional type of marker line is supported by DTSA-II: coincidence (sum) peak labels. Once the major elements in a spectrum have been identified and the characteristic lines labeled, DTSA-II can assist with identifying likely coincidence (sum) peaks. Selecting a narrow region of energies bounding the center of the unlabeled peak and then right-clicking over the yellow highlighted region will bring up a menu. Selecting the "ID sum peak" menu item will search through the list of selected major transitions for combinations whose sum falls within the selected energy window. The line will be labeled with the name of the transitions concatenated with a "+" sign or when the sum results from two photons from a single transition " $2 \times$ " the name of the transition.

\section{Publication Quality Output}

Generating publication-quality output of x-ray spectra can be tedious with generic plotting software. In addition to the challenges of importing $\mathrm{x}$-ray spectra data files, there are additional challenges related to labeling and scaling KLM markers, absorption edges, and escape peaks. DTSA-II provides two alternatives to save the current spectrum display in formats suitable for publication. The spectrum display window's main menu contains a "save" submenu. This submenu contains menu items to save the spectrum "as displayed" or "as a gnuPlot script." The "as displayed" option allows you to save the spectrum display in its current configuration as a Scalable
Vector Graphics (SVG), JPEG, PNG, TIFF, or Windows Bitmap (BMP) file. Select the format using the "file type" selector on the "Save as" dialog box. The resolution of this output is 4 times higher than that displayed on the screen while retaining the same aspect ratio. Fonts are also slightly upscaled to be more appropriate for publication. The SVG format is a vectorbased format, which means it can be scaled almost infinitely without pixelation. The SVG format can be edited using Adobe Illustrator [4], Corel Draw, or the open-source application InkScape [5], allowing additional labels to be added. The result from one of these programs can then be exported in a wide array of formats. The "save" $\rightarrow$ "as a gnuplot script" option outputs a script compatible with the open-source plotting package gnuplot [6]. Gnuplot is a flexible (albeit hard-to-use) package for generating publication-quality graphics in various formats including encapsulated Postscript (EPS) and LaTeX. DTSA-II will generate a starter script containing the spectral and marker line data, which can then be customized with a text editor.

\section{References}

[1] NWM Ritchie, Microscopy Today 19(1) (2011) 26-31.

[2] EMSA format: ISO 22029:2003 or http://www.amc.anl. gov/ANLSoftwareLibrary/EMMPDL(old)/Xeds/EMMFF/ emmff.doc.

[3] International Union of Pure and Applied Chemistry, "Nomenclature, symbols, units and their usage in spectrochemical analysis-VIII. Nomenclature system for X-ray spectroscopy (Recommendations 1991).” See Section 3. http://old.iupac.org/publications/analytical_compendium/ Cha10sec348.pdf

[4] Disclaimer: Certain commercial equipment, instruments, or materials are identified in this article to foster understanding. Such identification does not imply recommendation or endorsement by the National Institute of Standards and Technology, nor does it imply that the materials or equipment identified are necessarily the best available for the purpose.

[5] http://inkscape.org/

[6] http://www.gnuplot.info/

\section{Upgrade your Microscope!}

PIEZO STAGES, SCANNERS, MOTORS, ACTUATORS

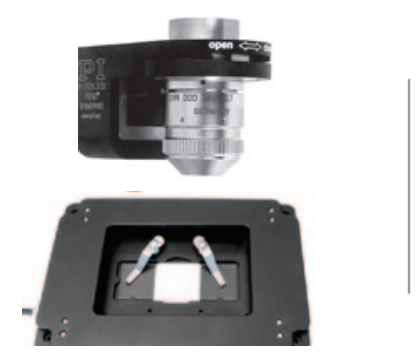

Piezo-Stages, Objective Scanners -I-Cost Effective Z, XY(Z) Stages - Very Fast: 5 msec Response

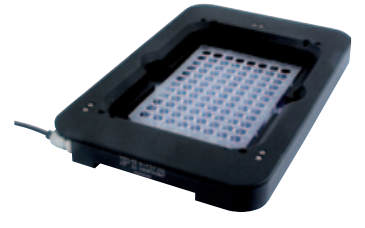

Well Plate Scanner

-1- High Speed \& Linearity - Travel to $500 \mu \mathrm{m}$

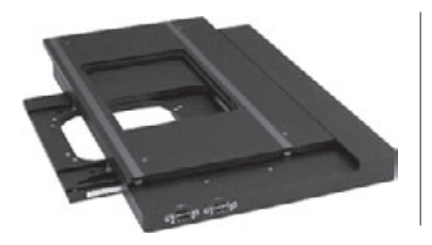

Ultra-Stable Piezo Motor Stages

- Self-Locking Ceramic Drive - Very Fast, Travel to $225 \times 85 \mathrm{~mm}$
19(1) (2011) 26-31.
http://www.amc.anl.
PDL(old)/Xeds/EMMFF/
Applied Chemistry,
and their usage in spectro-
clature system for X-ray
1991)." See Section 3.
analytical_compendium/
equipment, instru-
in this article to foster
PI (Physik Instrumente) LP
wo8.832.3456 info@pi-usa.us
usA Custom Design/Build
by the National Institute
does it imply that the
are necessarily the best

19(1) (2011) 26-31.
http://www.amc.anl.
PDL(old)/Xeds/EMMFF/
Applied Chemistry,
and their usage in spectro-
clature system for X-ray
1991)." See Section 3.
/analytical_compendium/
equipment, instru-
in this article to foster
po (Physik Instrumente) LP
wo8.832.3456 info@pi-usa.us
usA Custom Design/Build
by the National Institute
does it imply that the
are necessarily the best

19(1) (2011) 26-31.
http://www.amc.anl.
DL(old)/Xeds/EMMFF/
Applied Chemistry,
d their usage in spectro-
lature system for X-ray
1991)." See Section 3.
analytical_compendium/
in this article to foster
by the National Institute
does it imply that the
are necessarily the best
wsw.MicroscopeStage.net
usa Custom Design/Build
I

19(1) (2011) 26-31.
http://www.amc.anl.
DL(old)/Xeds/EMMFF/
Applied Chemistry,
d their usage in spectro-
lature system for X-ray
1991)." See Section 3.
analytical_compendium/
in this article to foster
by the National Institute
does it imply that the
are necessarily the best
wsw.MicroscopeStage.net
usa Custom Design/Build
I

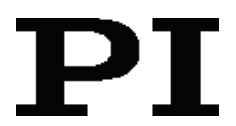

